\title{
Flow Rate through a Blood Vessel Deformed Due to a Uniform Pressure
}

\author{
Amy Cypher ${ }^{1}$, Mohamed B. Elgindi ${ }^{2}$, Hatem Kouriachi ${ }^{3}$, David Peschman ${ }^{4}$, Reba Shotwell ${ }^{5}$ \\ ${ }^{1}$ University of Wisconsin-Eau Claire, Eau Claire, USA; ${ }^{2}$ University of Wisconsin-Eau Claire and Texas A \& M University-Qatar, \\ Doha, Qatar; ${ }^{3}$ University of Wisconsin-Eau Claire, Eau Claire, USA; ${ }^{4}$ University of Wisconsin-River Falls, River Falls, USA; \\ ${ }^{5}$ University of Wisconsin-Madison, Madison, USA. \\ Email: elgindmb@uwec.edu, elgindmb@gmail.com
}

Received August 29 $9^{\text {th }}, 2011$; revised September $26^{\text {th }}, 2011$; accepted October $4^{\text {th }}, 2011$.

\begin{abstract}
In this paper, we present the mathematical equations that govern the deformation of an imbedded blood vessel under external uniform pressure taking into consideration the nonliner behavior of the soft tissue surrounding the vessel. We present a bifurcation analysis and give explicit formulas for the bifurcation points and the corresponding first order approximations for the non-trivial solutions. We then present the results of a MATLAB program that integrates the equilibrium equations and calculates the blood flow rate through a deformed cross section for given values of the elasticity parameters and pressure. Finally, we provide (numerical) verification that the flow rate as a function of the elasticity parameters of the soft tissue surrounding the blood vessel is convex, and therefore validate the invertibility of our model.
\end{abstract}

Keywords: Blood Vessel, Deformation of Elastic Tube

\section{Introduction}

Stability analysis for the buckling, post-buckling shapes and flow rate through an imbedded blood vessel under uniform external pressure were considered in [1]. In that paper, the soft tissues surrounding blood vessels are modeled by numerous linear independent springs. However, biological tissues are well known to respond in a nonlinear fashion to applied forces [2-6]. Since the support provided by the perivascular tissue is an important contributor to the in vivo structural stiffness of arteries, which will in turn affect the pressure-flow rate relationship, there is a critical need for further studies. In this paper, we examine the effect of replacing the linear spring in [1] by nonlinear ones on the post-buckling shapes and on the pressure-flow rate relationship. Furthermore, we verify (numerically) the convexity of the flow rate as a function of the elasticity parameters. This convexity of the (direct) problem is important to ensure its invertibility. That is, to ensure the solvability of the (more important) inverse problem, namely, to determine the elasticity parameters of the soft tissue surrounding the blood vessel from measurements of the deformation, the pressure, and the flow rate. The paper [1] assumes:

1) The tethering can be represented by numerous independent springs.

2) The springs are linear.
Motivated by the fact that biological tissues are known to respond in a nonlinear fashion to applied forces, we begin our series of studies to improve previous results by replacing Equation (8) of [1] by a nonlinear function $F=k_{1}(B C-A C)+k_{2} g(B C-A C)$. It is expected that this nonlinearity will have no effect on the stability analysis; however, it will alter the post-buckling shapes and flow rates through them. Interests in these postbuckling computations will make the present studies necessary and useful steps in the direction of describing tethered vessels more precisely. Furthermore, our (numerical) validation of the solvability of the inverse problem gives the simple physical model used in this project advantages over more complicated ones. The rest of this paper is organized in five sections. In Section 1, we define the variables and formulate the equilibrium equations. In Section 2, we give a bifurcation analysis of the equilibrium equations that lead to explicit formulas for the bifurcation points, dependent on spring stiffness, and the corresponding first order approximations for the bifurcation solutions. In Section 3, we show the numerical formulation [7]. In Section 4, we present our numerical results. In Section 5, we give some concluding remarks.

\section{Mathematical Formulation}

We consider the deformation of a thin-walled elastic 
cylinder tethered by continuously distributed nonlinear springs to a rigid outer cylinder (see Figure 1, below). The interior cylinder is subjected to internal pressure $P_{i}$, and external pressure $P_{e}$. This cylinder will remain circular until a bifurcation pressure difference is exceeded.

To formulate the mathematical equations governing the equlibrium, we consider the forces acting on an elemental length of the interior cylinder (see Figure 2, below).

In Figure 3, below, we analyze changes in the coordinates of an element length due to a displacement from point A to point $\mathrm{B}$.

\subsection{Notations}

In the rest of this paper, we use the following notations for our variables:
$P_{i}$ : Internal pressure
$P_{e}:$ External pressure
$S$ : Shearing force
$s^{\prime}:$ Arc length
$q_{t}^{\prime}:$ Tangential stress
$q_{n}^{\prime}:$ Normal stress
$M:$ Moment
$T:$ Tension
$\theta$ : Local curvature of vessel from $\mathrm{x}$ axis

\subsection{Assumptions}

We make the following assumptions:

1) The flow of blood through a tethered blood vessel is slow and steady.

2) The cross section does not vary much along a segment, so that the internal pressure is taken as constant (locally).

\subsection{Remark}

From the assumptions above, we conclude that we can solve for the deformed shape first, then calculate the flow rate afterward.

\subsection{Equilibrium Equations}

Balancing forces in the normal direction gives:

$$
\operatorname{Td} \theta=q_{n}^{\prime} d s^{\prime}+d S
$$

Balancing forces in tangential direction gives:

$$
q_{t}^{\prime} d s^{\prime}+S d \theta+d T=0
$$

Balance of moments gives:

$$
d M=S d s^{\prime}
$$

Assuming the wall thickness to be small compared to the radius, it follows that the moment $\mathrm{M}$ is proportional to the local curvature, where $\mathrm{E}$ and $\mathrm{I}$ are material constants [8]:

$$
M=E I \frac{\mathrm{d} \theta}{\mathrm{ds}}
$$

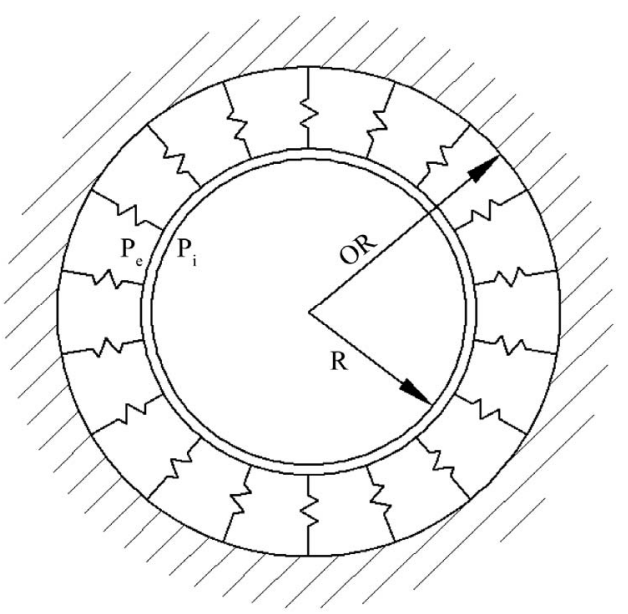

Figure 1. Elastic cylinder tethered to a rigid cylinder.

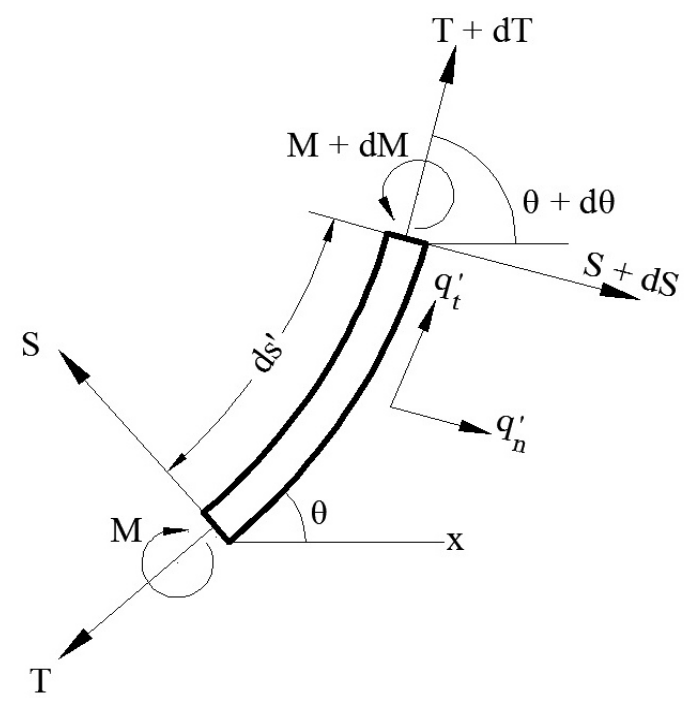

Figure 2. An element length.

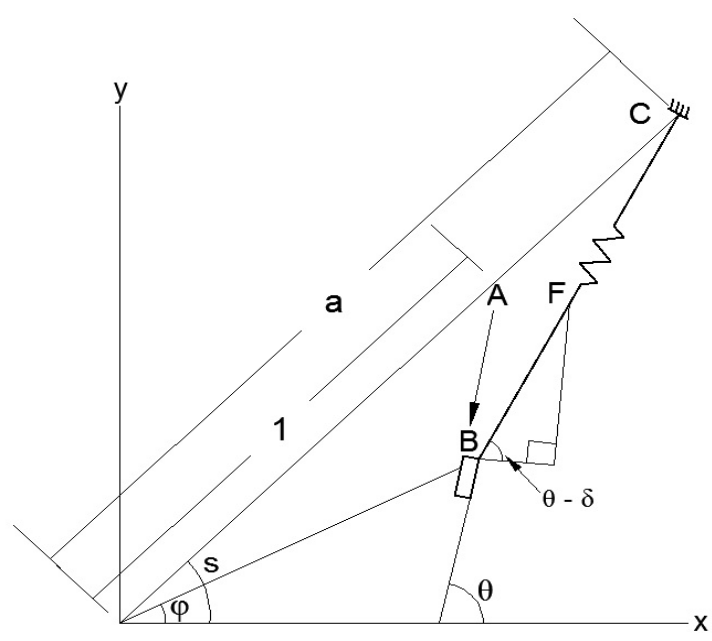

Figure 3. A small displacement from A to B. 
From which

$$
\frac{\mathrm{d} M}{\mathrm{~d} s^{\prime}}=E I \frac{\mathrm{d}^{2} \theta}{\left(\mathrm{d} s^{\prime}\right)^{2}}
$$

From (2) we get

$$
q_{t}^{\prime}+E I \frac{\mathrm{d}^{2} \theta}{\left(\mathrm{d} s^{\prime}\right)^{2}} \frac{\mathrm{d} \theta}{\mathrm{d} s^{\prime}}+\frac{\mathrm{d} T}{\mathrm{~d} s^{\prime}}=0
$$

From (1) we get

$$
T \frac{\mathrm{d} \theta}{\mathrm{d} s^{\prime}}-\frac{\mathrm{d} S}{\mathrm{~d} s^{\prime}}=q_{n}^{\prime}
$$

And this gives

$$
\frac{\mathrm{d} T}{\mathrm{~d} s} \frac{\mathrm{d} \theta}{\mathrm{d} s}+T \frac{\mathrm{d}^{2} \theta}{\mathrm{d} s^{2}}-\frac{\mathrm{d}^{2} S}{\mathrm{~d} s^{2}}=\frac{E I}{R^{2}} \frac{\mathrm{d} q_{n}}{\mathrm{~d} s}
$$

From (6) we get

$$
\frac{E I}{R^{2}} \frac{\mathrm{d} \theta}{\mathrm{d} s} q_{t}+\frac{E I}{R^{2}} \frac{\mathrm{d}^{2} \theta}{\mathrm{d} s^{2}}\left(\frac{\mathrm{d} \theta}{\mathrm{d} s}\right)^{2}+\frac{\mathrm{d} T}{\mathrm{~d} s} \frac{\mathrm{d} \theta}{\mathrm{d} s}=0
$$

From (8) and (9) we get

$$
\begin{aligned}
& \frac{\mathrm{d} \theta}{\mathrm{d} s} \frac{\mathrm{d}^{4} \theta}{\mathrm{d} s^{4}}-\frac{\mathrm{d}^{2} \theta}{\mathrm{d} s^{2}} \frac{\mathrm{d}^{3} \theta}{\mathrm{d} s^{3}}+\left[\left(\frac{\mathrm{d} \theta}{\mathrm{d} s}\right)^{3}-q_{n}\right] \frac{\mathrm{d}^{2} \theta}{\mathrm{d} s^{2}} \\
& +q_{t} \frac{\mathrm{d} \theta}{\mathrm{d} s^{2}}+\frac{\mathrm{d} q_{n}}{\mathrm{~d} s} \frac{\mathrm{d} \theta}{\mathrm{d} s}=0
\end{aligned}
$$

where

$$
\begin{aligned}
& s=\frac{s^{\prime}}{R} \\
& q_{n}=\frac{q_{n}^{\prime} R^{3}}{E I} \\
& q_{t}=\frac{q_{t}^{\prime} R^{3}}{E I}
\end{aligned}
$$

We also have

$$
\begin{aligned}
& \frac{d x}{d s}=\cos \theta \\
& \frac{d y}{d s}=\sin \theta
\end{aligned}
$$

Let $F_{0}$ be the tension per unit length per unit area of a spring at spring length of $a-1 \quad(a>1)$.

We define

$$
P=P_{e}-P_{i}-F_{0}
$$

We assume that the extra force due to deformation is $\mathrm{F}$ and is given by:

$$
F=k_{1}(B C-A C)+k_{2} g(B C-A C)
$$

where $k_{1}$ and $k_{2}$ are spring constants and $g$ is a nonlinear function.

$$
\begin{gathered}
A C=a-1 \\
B C=\sqrt{(a \cos s-x)^{2}+(a \sin s-y)^{2}}=d \\
\tan ^{2} x=\frac{\sin ^{2} x}{\cos ^{2} x}=\frac{1-\cos ^{2} x}{\cos ^{2} x}=\sec ^{2} x-1 \\
\sec ^{2}(\theta-\delta)=\tan ^{2}(\theta-\delta)+1=\left(\frac{a \sin s-y}{a \cos s-x}\right)^{2}+1 \\
=\frac{(a \sin s-y)^{2}+(a \cos s-x)^{2}}{(a \cos s-x)^{2}} \\
\cos ^{2}(\theta-\delta)=\frac{(a \cos s-x)^{2}}{(a \sin s-y)^{2}+(a \cos s-x)^{2}} \\
=\frac{(a \cos s-x)^{2}}{d^{2}} \\
\cos (\theta-\delta)=\frac{a \cos s-x}{d} \\
\sin (\theta-\delta)=\frac{a \sin s-y}{d}
\end{gathered}
$$

But

$$
\begin{gathered}
\cos \theta \cos (\theta-\delta)+\sin \theta \sin (\theta-\delta) \\
=\cos (\theta-\theta+\delta)=\cos \delta \\
\Rightarrow \cos \theta \frac{a \cos s-x}{d}+\sin \theta \frac{a \sin s-y}{d}=\cos \delta \\
q_{t}=\frac{F(Z)}{d}[\cos \theta(a \cos s-x)+\sin \theta(a \sin s-y)] \\
q_{n}=\frac{F(Z)}{d}[\sin \theta(a \cos s-x)+\cos \theta(a \sin s-y)] \\
F(Z)=k_{1}(Z)+k_{2} g(Z) \\
Z=d-a+1
\end{gathered}
$$

\section{Bifurcation Analysis}

For low values of the pressure difference, the interior cylinder remains circular. As the pressure difference increases byond some critical values, non-circular solutions occur (see Figure 4, below). These critical values of the pressure difference are called bifurcation points while the corresponding non-circular solutions are called bifurcation solutions. It is well known that bifurcation may occur only at pressure difference values that correspond to a singular linearized problem about the circular solution. 


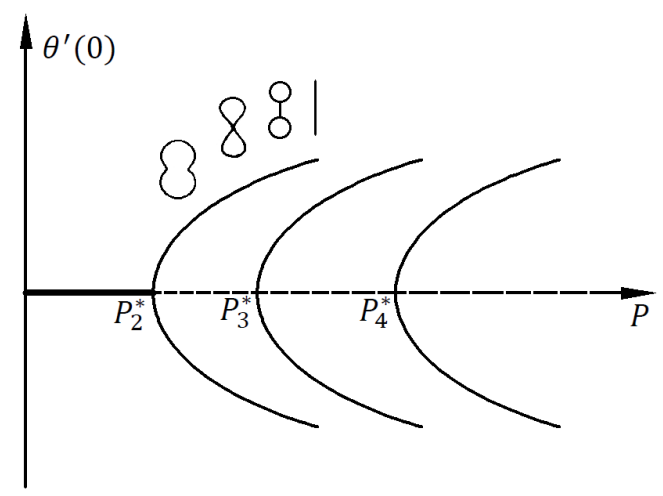

Figure 4. Bifurcation points with solutions for $N=2,3$, and 4 .

In this section, we present the calculations to find the bifurcation points: the critical values of the pressure difference at which the vessel deforms into non-circular shapes.

First, we set

$$
\begin{aligned}
& y_{1}(s) \equiv \theta \quad \text { (local angle) } \\
& y_{2}(s) \equiv \text { Moment } \\
& y_{3}(s) \equiv \text { Shear } \\
& y_{4}(s) \equiv \text { Tension } \\
& y_{5}(s) \equiv x \\
& y_{6}(s) \equiv y
\end{aligned}
$$

where $s \in\left[0, \frac{2 \pi}{N}\right]$ is the arclength.

We define the state vector:

$$
Y(s)=\left[y_{1}(s), y_{2}(s), y_{3}(s), y_{4}(s), y_{5}(s), y_{6}(s)\right]
$$

Then the equations of equilibrium can be written as:

$$
Y^{\prime}(s)=F(Y, P)=\left[\begin{array}{c}
y_{2} \\
y_{3} \\
y_{4} y_{2}-q_{n}-P \\
-y_{3} y_{2}-q_{t} \\
\cos y_{1} \\
\sin y_{1}
\end{array}\right]=0
$$

with boundary conditions:

$$
\begin{gathered}
y_{1}(0)=\frac{\pi}{2} \\
y_{3}(0)=y_{6}(0)=0 \\
y_{1}\left(\frac{2 \pi}{N}\right)=\frac{\pi}{2}+\frac{2 \pi}{N} \\
y_{3}\left(\frac{2 \pi}{N}\right)=0
\end{gathered}
$$

$$
y_{5}^{2}(0)+y_{6}^{2}(0)=y_{5}^{2}\left(\frac{2 \pi}{N}\right)+y_{6}^{2}\left(\frac{2 \pi}{N}\right)
$$

$P, q_{n}$, and $q_{t}$ defined by:

$P=$ Pressure difference,

$$
\begin{aligned}
& q_{n}=\frac{F(Z)}{d}\left[\sin y_{1}\left(a \cos s-y_{5}\right)-\cos y_{1}\left(a \sin s-y_{6}\right)\right], \\
& q_{t}=\frac{F(Z)}{d}\left[\cos y_{1}\left(a \cos s-y_{5}\right)-\sin y_{1}\left(a \sin s-y_{6}\right)\right],
\end{aligned}
$$

where $q_{n}$ and $q_{t}$ are the normal and tangential components of stress per unit length, $F(Z)$ is the force due to the springs, which is given by:

$$
F(Z)=k_{1} Z+k_{2} g(Z)
$$

where

And

$$
Z=d-a+1
$$

$$
d=\sqrt{\left(a \cos s-y_{5}\right)^{2}+\left(a \sin s-y_{6}\right)^{2}} .
$$

In the circular case, the Basic Solution is given by:

$$
Y_{0}(s)=\left[\begin{array}{c}
y_{1}(s) \\
y_{2}(s) \\
y_{3}(s) \\
y_{4}(s) \\
y_{5}(s) \\
y_{6}(s)
\end{array}\right]=\left[\begin{array}{c}
s+\frac{\pi}{2} \\
1 \\
0 \\
-P \\
\cos s \\
\sin s
\end{array}\right]
$$

where $d=a-1, Z=0, q_{t}=0$, and $q_{n}=g(0)$.

Assuming that $g(0)=0$, we have $Y_{0}^{\prime}(s)=F\left(Y_{0}, P\right)$, $\forall P$, and furthermore $Y_{0}(s)$ satisfies the boundary conditions for formula (31). The Fréchet derivative of $F(Y, P)$ at the basic solution is given by:

$$
F_{Y}\left(Y_{0}\right)=\left[\begin{array}{cccccc}
0 & 1 & 0 & 0 & 0 & 0 \\
0 & 0 & 1 & 0 & 0 & 0 \\
-\frac{\partial q_{n}}{\partial y_{1}} & y_{4} & 0 & y_{2} & -\frac{\partial q_{n}}{\partial y_{5}} & -\frac{\partial q_{n}}{\partial y_{6}} \\
-\frac{\partial q_{t}}{\partial y_{1}} & -y_{3} & -y_{2} & 0 & -\frac{\partial q_{t}}{\partial y_{5}} & -\frac{\partial q_{t}}{\partial y_{6}} \\
-\sin y_{1} & 0 & 0 & 0 & 0 & 0 \\
\cos y_{1} & 0 & 0 & 0 & 0 & 0
\end{array}\right]
$$

where:

$$
\begin{gathered}
\left.\frac{\partial q_{n}}{\partial y_{1}}\right|_{Y=Y_{0}}=\left.\frac{\partial q_{t}}{\partial y_{1}}\right|_{Y_{0}}=\left.\frac{\partial q_{t}}{\partial y_{5}}\right|_{Y_{0}}=\left.\frac{\partial q_{t}}{\partial y_{6}}\right|_{Y_{0}}=0 \\
\left.\frac{\partial q_{n}}{\partial y_{5}}\right|_{Y_{0}}=-k_{1} \cos s
\end{gathered}
$$




$$
\left.\frac{\partial q_{n}}{\partial y_{6}}\right|_{Y_{0}}=-k_{1} \sin s
$$

Therefore, the linearized problem about the basic solution $Y_{0}(s)$ is:

$Y^{\prime}(s)$

$$
=\left[\begin{array}{cccccc}
0 & 1 & 0 & 0 & 0 & 0 \\
0 & 0 & 1 & 0 & 0 & 0 \\
0 & -P & 0 & 1 & k_{1} \cos s & k_{1} \sin s \\
0 & 0 & -1 & 0 & 0 & 0 \\
-\cos s & 0 & 0 & 0 & 0 & 0 \\
-\sin s & 0 & 0 & 0 & 0 & 0
\end{array}\right]\left[\begin{array}{l}
y_{1}(s) \\
y_{2}(s) \\
y_{3}(s) \\
y_{4}(s) \\
y_{5}(s) \\
y_{6}(s)
\end{array}\right](40)
$$

Which may be written as:

$$
y_{1^{\prime \prime \prime}}=-(P+1) y_{1^{\prime \prime}}+k_{1} \frac{\mathrm{d}}{\mathrm{d} s}\left(y_{5} \cos s+y_{6} \sin s\right)
$$

Integrating twice gives:

$$
y_{1^{\prime \prime}}+(P+1) y_{1}-k_{1} \int y_{5} \cos s+y_{6} \sin s \mathrm{~d} s=c_{1} s+c_{2}
$$

with boundary conditions:

$$
\begin{gathered}
y_{1}(0)=y_{3}(0)=y_{6}(0)=0 \\
y_{1}\left(\frac{2 \pi}{N}\right)=y_{3}\left(\frac{2 \pi}{N}\right)=0 \\
y_{5}(0)=\cos \left(\frac{2 \pi}{N}\right) y_{5}\left(\frac{2 \pi}{N}\right)+\sin \left(\frac{2 \pi}{N}\right) y_{6}\left(\frac{2 \pi}{N}\right)
\end{gathered}
$$

Therefore, $c_{2}=0$.

To solve the differential equation:

$$
y_{1^{\prime \prime}}+(P+1) y_{1}-k_{1}\left(y_{5} \sin s+y_{6} \cos s\right)=c_{1} s
$$

We write the solution in the form:

$$
\begin{gathered}
y_{1}=\theta=\left(s+\frac{\pi}{2}\right)+\sum_{n=1}^{\infty} b_{n} \sin n N s \\
y_{5}=x=\cos s+\sum_{n=1}^{\infty} b_{n}\left[\frac{s \cos n N-1}{n N-1}+\frac{s \cos n N+1}{n N-1}\right]+c_{3} \\
y_{6}=y=\sin s-\sum_{n=1}^{\infty} \frac{b_{n}}{2}\left[\frac{s \sin n N-1}{n N-1}-\frac{s \sin n N+1}{n N-1}\right]+c_{4}
\end{gathered}
$$

where $N=2,3,4, \cdots$.

Substituting these equations into the differential equation gives:

$$
\sum_{n=1}^{\infty} b_{n} \sin n N s\left(-n^{2} N^{2}+P+1-\frac{k_{1}}{n^{2} N^{2}-1}\right)=0
$$

Then

$$
b_{n}\left(-n^{2} N^{2}+P+1-\frac{k_{1}}{n^{2} N^{2}-1}\right)=0, \forall n>1
$$

For a non-zero solution (a bifurcation point) to exist, $b_{1}$ must be $\neq 0$.

Therefore,

$$
-N^{2}+P+1-\frac{k_{1}}{N^{2}-1}=0
$$

From which we get:

$$
P_{N}^{*}=N^{2}-1+\frac{k_{1}}{N^{2}-1}
$$

where $P_{N}^{*}$ is the critical pressure value, or bifurcation point, at which the vessel deforms into a shape with $\mathrm{N}$ axes of symmetry.

This result allows us to find the bifurcation points for given values of $N$ and $k_{1}$. Before the first bifurcation point, for $P \in\left[0, P_{2}^{*}\right)$, we have the basic (trivial) solution which corresponds to the undeformed shape. The deformed shapes corresponding to $N=2,3,4, \cdots$, $N$ exist for $P \geq P_{2}^{*}$, where the circular solution becomes unstable, and uniqueness is lost. A series expansion of the first order approximations of the bifurcation solutions are given by formula (43).

Additionally, equating $P_{N}^{*}$ with $P_{N+1}^{*}$ gives us the pressure where the shape with $N$ axes of symmetry collapses. For example, the $N=2$ case occurs for $0 \leq$ $k_{1} \leq 24$, while the $N=3$ case occurs for $24 \leq k_{1} \leq 120$.

\section{Numerical Formulation}

Due to the assumed uniform external pressure, the vessel will deform into radially symmetric shapes, with $\mathrm{N}$ axes of symmetry ( $N$, an integer, $\geq 2$ ).

Given $P, a, N, k_{1}$, and $k_{2}$, we write the equilibrium equations in the form:

$$
\begin{aligned}
& y_{1}^{\prime}=y_{2} \\
& y_{2}^{\prime}=y_{3} \\
& y_{3}^{\prime}=y_{4} \\
& y_{4}^{\prime}=\frac{y_{3} y_{2}}{y_{2}}-\frac{y_{3}}{y_{2}}\left(y_{2}^{3}-q_{n}\right)-q_{t} y_{2}-q_{n}^{\prime} \\
& y_{5}^{\prime}=\cos y_{1} \\
& y_{6}^{\prime}=\sin y_{1}
\end{aligned}
$$

where

$y_{1}=\theta, \quad y_{2}=\theta^{\prime}, \quad y_{3}=\theta^{\prime \prime}, \quad y_{4}=\theta^{\prime \prime \prime}, \quad y_{5}=x, \quad y_{6}=y$ and

$$
\begin{aligned}
& q_{t}=\frac{F(Z)}{d}\left[\cos \theta\left(a \cos s-y_{5}\right)+\sin \theta\left(a \sin s-y_{6}\right)\right](51) \\
& q_{n}=\frac{F(Z)}{d}\left[\sin \theta\left(a \cos s-y_{5}\right)+\cos \theta\left(a \sin s-y_{6}\right)\right](52)
\end{aligned}
$$




$$
\begin{gathered}
d=\sqrt{\left(a \cos s-y_{5}\right)^{2}+\left(a \sin s-y_{6}\right)^{2}} \\
Z=d-a+1
\end{gathered}
$$

and $s \in\left[0, \frac{2 \pi}{N}\right]$, with boundary conditions

$$
\begin{aligned}
& y_{1}(0)-\frac{\pi}{2}=0 \\
& y_{3}(0)=0 \\
& y_{6}(0)=0 \\
& y_{1}\left(\frac{2 \pi}{N}\right)-\left(\frac{\pi}{2}+\frac{2 \pi}{N}\right)=0 \\
& y_{3}\left(\frac{2 \pi}{N}\right)=0 \\
& \left(y_{5}^{2}(0)-y_{6}^{2}(0)\right)-\left(y_{5}^{2}\left(\frac{2 \pi}{N}\right)-y_{6}^{2}\left(\frac{2 \pi}{N}\right)\right)=0 .
\end{aligned}
$$

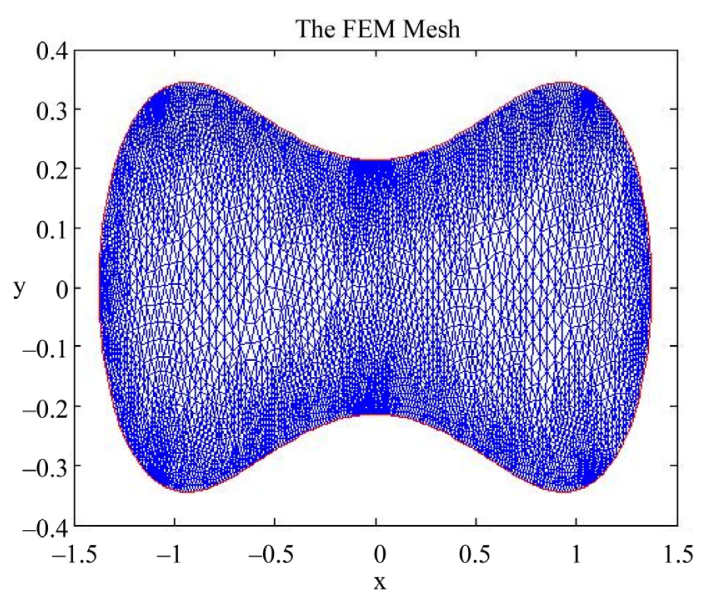

We can then numerically solve for the shape of the deformed vessel. Using this shape, we solve the following (normalized) Poisson equation $\nabla v=-1$ in a MATLAB program to find the velocity of the blood, $v(x, y)$. We can then find the flow rate through the deformed vessel by integrating the velocity function over the area of the cross sectional area of the vessel.

\section{Numerical Results}

We created a MATLAB code that uses $a, k_{1}, k_{2}, N$, and $P$ as inputs to solve for the shape and flow rate. The figures below are example outputs of this program for the cases $N=2,3$, and 4 . We used the following model to represent the nonlinearality of the soft tissue surrounding the blood vessel:

$$
F(Z)=k_{1} Z+k_{2} \tanh (Z)
$$

In each pair below, the figure on the left shows the

Figure 5. MATLAB results for $N=2$.
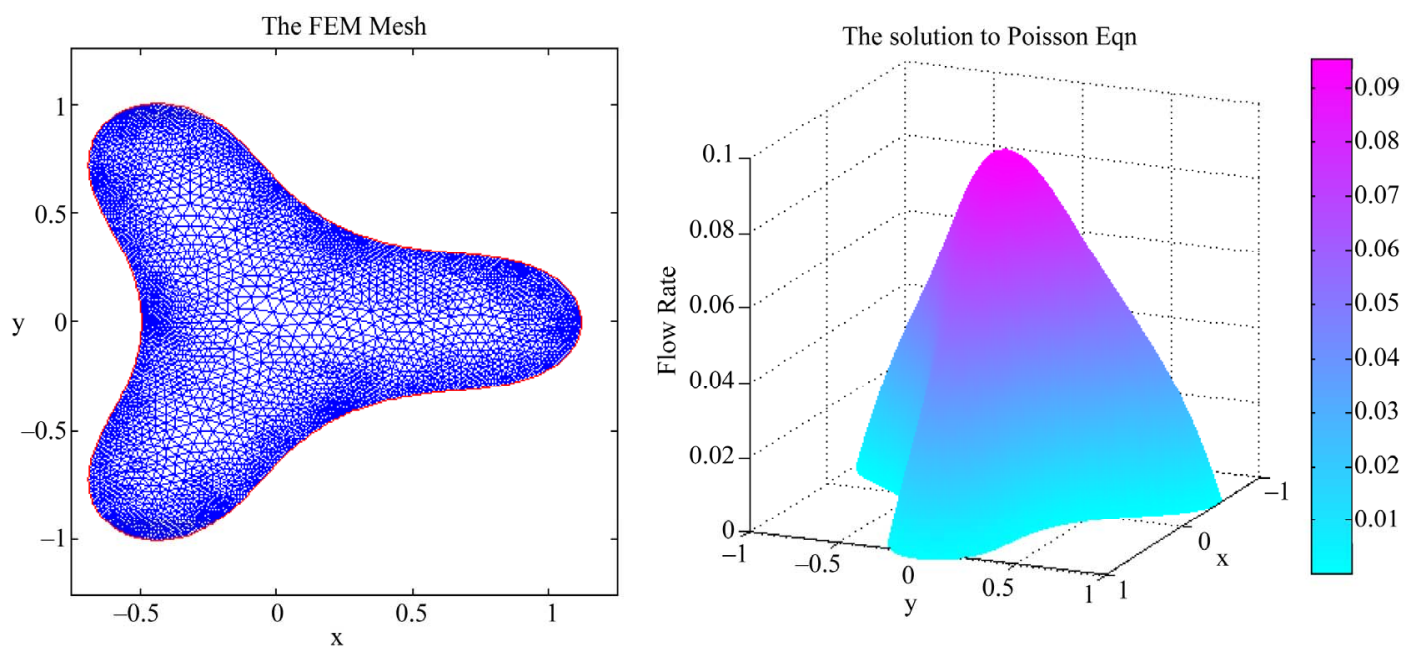

Figure 6. MATLAB results for $N=3$. 

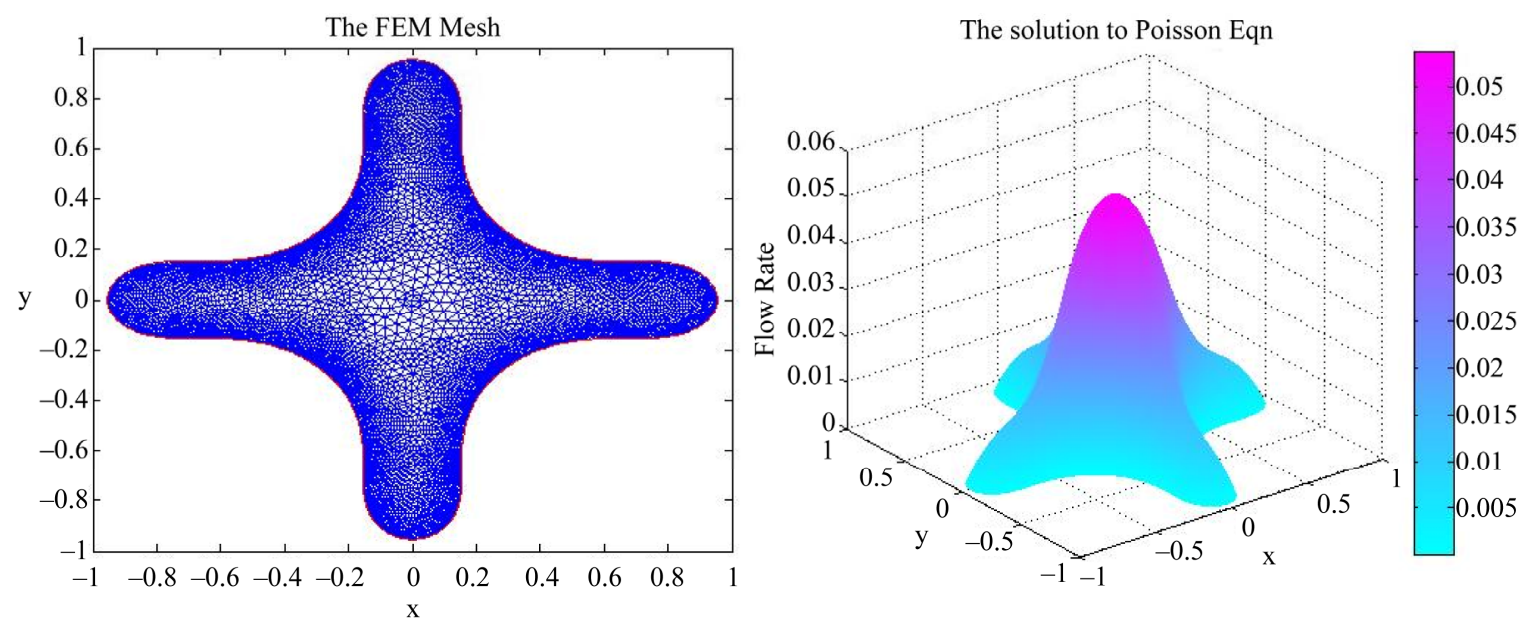

Figure 7. MATLAB results for $N=4$.

shape of the deformed vessel, which is then meshed to create the image on the right, a $3 D$ representation of the velocity profile of the blood flowing through the vessel.

\section{Conclusions}

In real situations, the pressure and deformed shape can be easily determined using medical technology (for example, by x-ray or ultrasound), hence we would seek to determine the elasticity of the tissue $\left(k_{1}\right.$ and $\left.k_{2}\right)$ based on a given pressure and shape, since those can
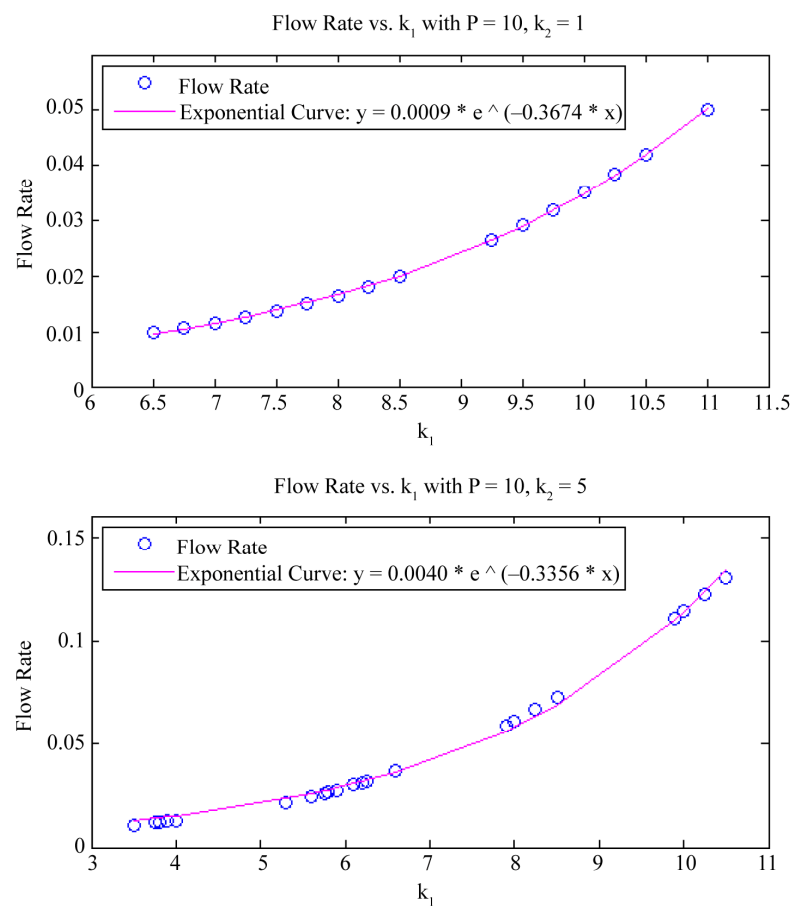

only be determined while the tissue is in vivo.

The tables of graphs below show the varying of $k_{1}$ and $k_{2}$ independently with a constant pressure and a symmetry shape of $N=2$. As can be seen, all graphs show a strictly increasing relationship. By combining the flow rate versus $k_{1}$ and $k_{2}$ data, we created a $3 D$ model that also shows convex curvature. This model verifies (numerically) that a unique minimum can be obtained. The unique minimum could then be determined by using Newton's Method, giving us a way to determine the elasticity parameters, $k_{1}$ and $k_{2}$.
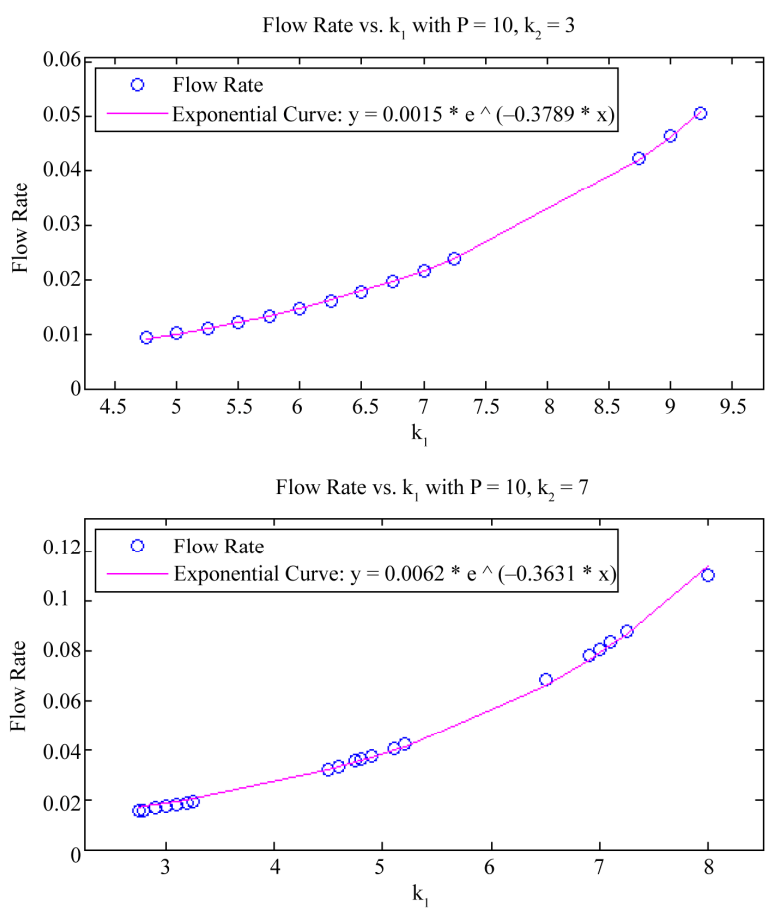

Figure 8. $N=2$ graphs of flow rate and $k_{1}$ with constant $k_{2}$ and pressure. 

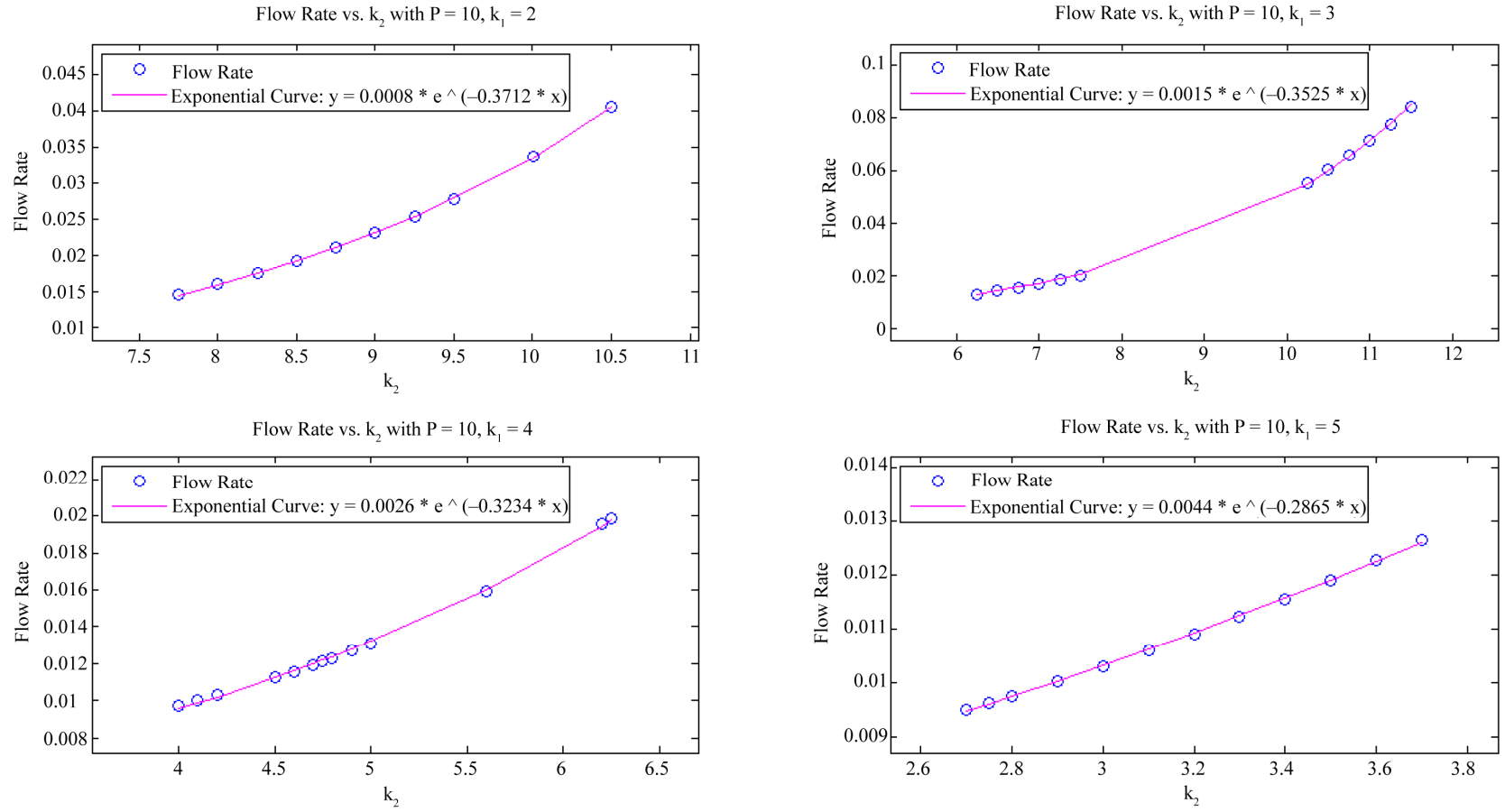

Figure 9. $N=2$ graphs of flow rate and $k_{2}$ with constant $k_{1}$ and pressure.

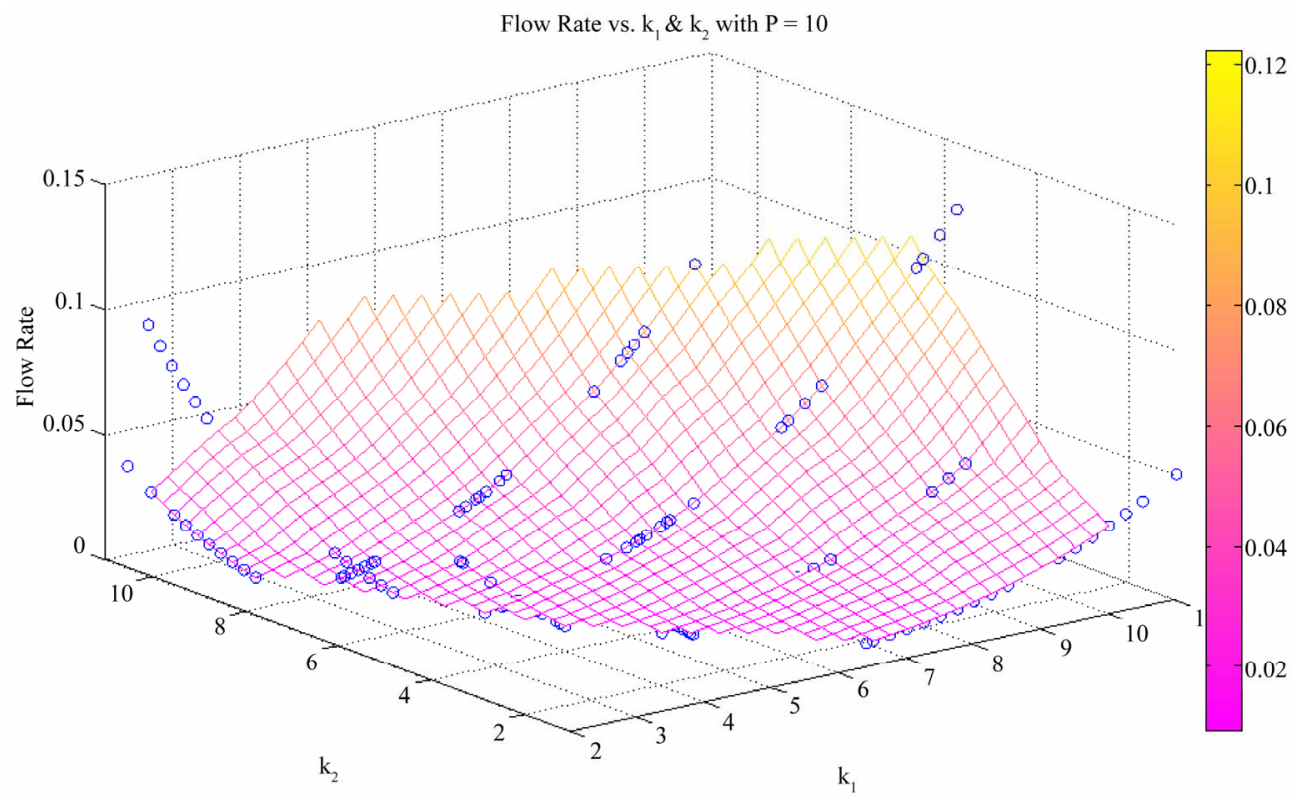

Figure 10. 3D graph of flow rate, $k_{1}$, and $k_{2}$ with constant pressure.

\section{Acknowledgements}

National Science Foundation grant number 0552350 and UW-Eau Claire Reasearch Office for their support of the SUREPAM program.

\section{REFERENCES}

[1] C. Y. Wang, L. T. Watson and M. P. Kamat, "Buckling,
Postbuckling, and Flow Rate Through a Tethered Elastic Cylinder Under External Pressure," Journal of Applied Mechanics, Vol. 50, No. 1, pp. 13-18, 1983. doi:10.1115/1.3166981

[2] D. H. Bergel, "The Properties of Blood Vessels," In: Biomechanics, Its Foundations and Objectives, Eds., Prentice-Hall, Englewood, 1972.

[3] Y. C. Fung, "Biomechanics, Mechanical Properties of 
Living Tissues," Springer-Verlag, New York, 1990.

[4] A. C. Guyton and J. E. Hall, "Textbook of Medical Physiology," Saunders Company, 1996.

[5] A. H. Moreno, A. I. Katz, L. D. Gold and R. V. Reddy, "Mechanics of Distension of Dog Veins and Other Very Thin-Walled Tubular Sturctures," Circulation Research, Vol. 27, No. 6, 1970, pp. 1069-1080.

[6] K. Osterloch, P. Gaehtgens, and A. Pries, "Determination of Microvascular Flow Pattern Formation in Vivo," Ame- rican Journal of Botany, Vol. 278, No. 4, 2000, pp. H1142-H1152.

[7] J. Stoer and R. Bulirsch, "Introduction to Numerical Analysis," Springer-Verlag, New York, 1980.

[8] M. B. M. Elgindi, D. H. Y. Yen and C. Y. Wang, "Deformation of a Thin-Walled Cylindrical Tube Submerged in a Liquid," Journal of Fluids and Structure, Vol. 6, No. 3, 1992, pp. 353-370. doi:10.1016/0889-9746(92)90014-T 